\title{
Ring Resonators with Sagnac Loops for Photonic Processing in DWDM Backbone Networks
}

\author{
Carmen Vázquez, Julio Montalvo, Pedro C. Lallana \\ Displays and Photonics Applications Group, Department of Electronics Technology \\ Carlos III University of Madrid, 30 Universidad Avenue, 28911, Leganés (Madrid). SPAIN
}

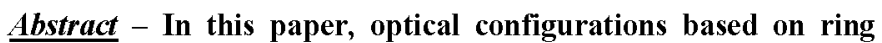
resonators (RR) with an internal Sagnac (SG) loop in the feedback path, are analyzed in terms of their amplitude response and dispersive properties for filtering and chromatic dispersion managing in digital transmission systems over amplified singlemode fiber (SMF) spans in DWDM backbone networks. Design issues for the architecture as regards quadratic dispersion and magnitude distortion are provided. The $R \mathbf{R}+\mathbf{S G}$ compound filter provides frequency tunability of the amplitude and dispersion peaks by adjusting a coupling coefficient of an optical coupler, with no need for using integrated thermo-optic nor currentinjection based phase shifters. The configuration can be employed as an additional structure for a general RR-based design and synthesis architecture, allowing bandwidth increase of dispersion compensators and flexibility. The performance of a compound filter consisting of a two $R R$ in series stage and a RR+SG filter are reported as a more compact and effective solution for existing multi-channel SMF backbone links operating at high bit rates. Design guidelines of an integrated ring resonator based on polymer technology for showing feasibility of the proposal is reported.
\end{abstract}

$\underline{\text { Keywords }}$ - BER, dispersion compensation, ring resonator, Sagnac interferometer, tunable filters.

\section{INTRODUCTION}

Nowadays ring resonators (RR) are widely used for photonic processing in optical communication systems. RR have applications as routers in Self-Routing Frequency Division Multiple Access (SR-FDMA) networks $[1,2]$, to inform the destination node that a packet has been transmitted and to which channel to tune its receiver [3], and for performance monitoring. They have also been used as filters [4] in Optical Frequency Division Multiplexing transmission systems (OFDM) [5], or in DWDM (Dense Wavelength Division Multiplexing) systems with carriers spacing of 50 GHz, or less [6]. They have also been used as chromatic dispersion compensating filters in high bit rate digital transmission systems [7], in lasers and in non-linear optical applications such as all-optical switches $[8,9]$.

Different technologies are reported for filtering and chromatic dispersion compensation, such as Fabry-Perot (FP), Planar lightwave circuits (PLC), Mach-Zehnder interferometers (MZI), arrayed waveguide gratings (AWG), thin-film dielectric interference filters (TTF), and conventional fiber Bragg gratings (FBG). General filter design techniques based on signal processing approaches are reported in [10] using compound lattice architectures with MZI and RR, including all-pass filters for dispersion control applications. More recently, tunable dispersion control with micro-electro-mecanical (MEM) actuated all-pass filter [11] was fabricated.

On the other hand, the analysis of a Sagnac (SG) loop in ring resonators (RR) has been reported as a tuneable optical filter [12-13] with ultra-narrow-bandwidth for use in Dense WDM systems. This proposal allows tunability achievement just by adjusting a coupling coefficient of an optical coupler, with no need for using integrated thermo-optic, nor currentinjection based phase shifters.

In this paper, we focus on the filtering and dispersive properties of the RR with SG loop filter, for among others chromatic dispersion managing in digital transmission systems over amplified single-mode fiber (SMF) spans in DWDM backbone networks. In section 2 the transfer function is analyzed and design issues for the architecture are provided, section 3 is devoted to in line response and design issues in polymeric technology are reported in section 4.

\section{FILTER ARCHITECTURE}

The schematic of a RR with internal SG filter [12] is shown in Fig. 1, being $K_{1}, K_{2}$ the cross-coupling power factors, $\gamma_{1}, \gamma_{2}$ the corresponding power excess losses of the couplers, $T x, T y$ the power transmission of the corresponding feedback segments per roundtrip, and $L_{t}$ the total feedback loop length. The diagram in Fig. 1(b) shows the mirror 
equivalent model of the filter considering unidirectional propagation of the light, which makes easier the analysis of the structure. Considering this mirror model, the structure can be analyzed as an unidirectional ring resonator with an internal transfer function dependent on the Sagnac $2 \times 2$ matrix parameters.

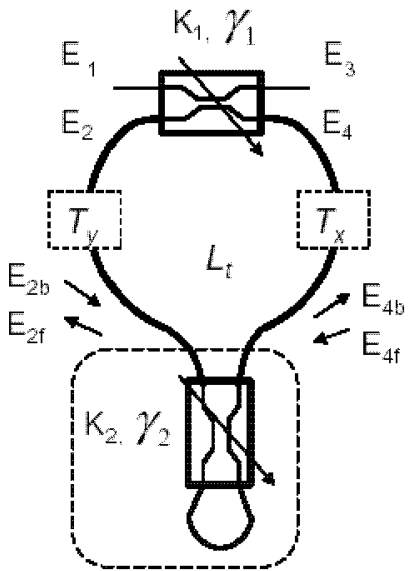

Sagnac loop

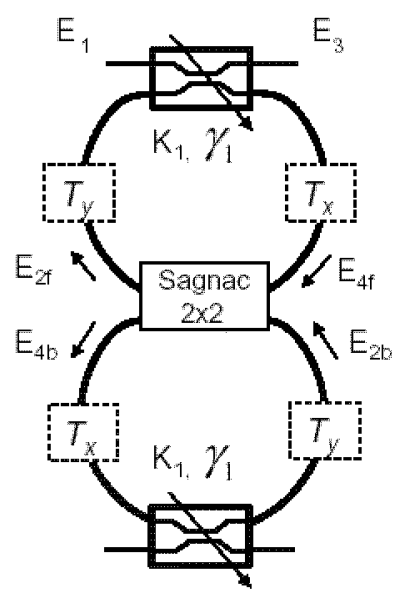

Fig. 1. Photonic schematic of the $\mathrm{RR}+\mathrm{SG}$ filter (left) and its mirror equivalent model (right).

\section{A. Analysis of the RR $+S G$ filter in the Z-transform domain}

The scattering matrix of the optical coupler and the Sagnac $2 \times 2$ module, named $M$ and $S$, respectively, are defined as follows [12]:

$$
\begin{gathered}
(M)=\left(1-\gamma_{1}\right)^{1 / 2} \cdot\left(\begin{array}{ll}
\sqrt{1-K_{1}} & -j \sqrt{K_{1}} \\
-j \sqrt{K_{1}} & \sqrt{1-K_{1}}
\end{array}\right) \\
(S)=\left(\begin{array}{ll}
F T & F R \\
F R & F T
\end{array}\right)
\end{gathered}
$$

where:

$$
\begin{gathered}
F T=\left(1-\gamma_{2}\right) \cdot\left(1-2 \cdot K_{2}\right) \\
F R=2 \cdot j \cdot\left(1-\gamma_{2}\right) \cdot \sqrt{K_{2} \cdot\left(1-K_{2}\right)}
\end{gathered}
$$

Thus the relationships between ports are:

$$
\begin{aligned}
& \left(\begin{array}{l}
E_{3} \\
E_{4}
\end{array}\right)=(M) \cdot\left(\begin{array}{l}
E_{1} \\
E_{2}
\end{array}\right) \\
& \left(\begin{array}{l}
E_{2 f} \\
E_{4 b}
\end{array}\right)=(S) \cdot\left(\begin{array}{l}
E_{4 f} \\
E_{2 b}
\end{array}\right)
\end{aligned}
$$

For notation reasons, from now on we will consider $T x=T y$, which is a realistic situation considering that the
$\mathrm{RR}+\mathrm{SG}$ configuration is symmetrical, and will use the additional variable $g$, defined as:

$$
g \equiv T x=T y
$$

The Z-transform of the transfer function of the SG with feedback in the mirror model, $h(z)$, has the following expression:

$$
h(z) \equiv \frac{E_{2 f}}{E_{4 f}}=\frac{F T-\left(F T^{2}-F R^{2}\right) \cdot T^{*} \cdot z^{-1}}{1-F T \cdot T^{*} \cdot z^{-1}}
$$

where:

$$
T^{*}=g \cdot\left(1-\gamma_{1}\right)^{1 / 2} \cdot\left(1-K_{1}\right)^{1 / 2}
$$

A first approach to the transfer function of the compound structure in the Z-Transform domain is the following:

$$
H(z) \equiv \frac{E_{3}}{E_{1}}=\frac{\rho-\left(1-\gamma_{1}\right) \cdot h^{*}(z) \cdot z^{-1}}{1-\rho \cdot h^{*}(z) \cdot z^{-1}}
$$

being:

$$
\begin{gathered}
\rho=\left(1-\gamma_{1}\right)^{1 / 2} \cdot\left(1-K_{1}\right)^{1 / 2} \\
h^{*}(z)=g \cdot h(z)
\end{gathered}
$$

\section{B. Magnitude response and resonance conditions for filtering}

The complete Z-transform of the architecture can be obtained by using (8) and (12) in (10); then the zeros and poles of the transfer function can be obtained by factorizing numerator and denominator of the final expression:

$$
H(z)=\rho \cdot \prod_{i=1}^{2} \frac{\left(1-c_{i} \cdot z^{-1}\right)}{\left(1-p_{i} \cdot z^{-1}\right)}
$$

obtaining:

$$
\begin{gathered}
c_{i}=\frac{g \cdot\left(1-\gamma_{1}\right)^{1 / 2}}{2 \cdot\left(1-K_{1}\right)^{1 / 2}} \cdot\left[F T \cdot\left(2-K_{1}\right)+\right. \\
\left.+(j)^{2(i-1)} \cdot \sqrt{F T^{2} \cdot K_{1}^{2}+4 \cdot\left(1-K_{1}\right) \cdot F R^{2}}\right] \\
p_{i}=g \cdot\left[\left(1-\gamma_{1}\right) \cdot\left(1-K_{1}\right)\right]^{1 / 2} . \\
\cdot\left[F T+(j)^{2(i-1)} \cdot F R\right]
\end{gathered}
$$

For designing a selective or notch tunable filter, complex conjugate poles have to be considered [12] and the conditions to be fulfilled are reported in Table I. 
TABLE I.

RESONANCE CONDITIONS AT P P FoR COMPLEX CONJUGATE POLES

\begin{tabular}{|l}
\hline \multicolumn{1}{|c}{ RR with SG filter } \\
\hline Maxima condition
\end{tabular}

$$
\begin{aligned}
& g=\frac{1}{\left(1-\gamma_{1}\right)^{1 / 2}\left(1-\gamma_{2}\right)\left(1-K_{1}\right) e^{-\alpha L_{T}}} \\
& \text { Maxima frequencies }{ }^{\mathrm{a}} \\
& 2 \pi k=\frac{2 \pi f_{1,2} n_{e f}}{c} L_{T} \mp \phi_{s} \\
& g=\frac{1}{\left(1-\gamma_{1}\right)^{1 / 2}\left(1-\gamma_{2}\right) e^{-\alpha L_{T}}} \\
& 2 \pi k=\frac{2 \pi f_{1,2} n_{e f}}{c} L_{T} \mp \phi_{s c}
\end{aligned}
$$

the waveguides

Those equations are valid for the $K_{1}$ and $K_{2}$ values satisfying the equation:

$$
K_{1}<\frac{-1+\sqrt{1+4 A}}{2 A}
$$

being:

$$
A=\frac{K_{2}\left(1-K_{2}\right)}{\left(1-2 K_{2}\right)^{2}}
$$

Under that condition, the values of the phases are given by:

$$
\phi_{s}=\tan ^{-1}\left[\frac{\left(2-K_{1}\right) \sqrt{K_{2}\left(1-K_{2}\right)}}{\sqrt{\left(1-K_{1}\right)\left(1-2 K_{2}\right)^{2}-K_{1}^{2}\left(K_{2}-K_{2}^{2}\right)}}\right]
$$

$\phi_{s c}=\arg \left(\frac{\sqrt{-K_{1}^{2}\left(1-2 K_{2}\right)^{2}+16\left(1-K_{1}\right)\left(1-K_{2}\right) K_{2}}}{\left(2-K_{1}\right)\left(1-2 K_{2}\right)}\right)$

\section{Quadratic dispersion and magnitude distortion of the $R R+S G$ filter for chromatic dispersion compensation}

From the expression in (13) the normalized group delay of the filter for $z=\exp (j \cdot \Omega)$ can be obtained as follows:

$$
\begin{aligned}
\tau_{n}= & \sum_{j=1}^{N} \frac{\left|p_{j}\right| \cdot\left(\cos \left(\Omega-\phi_{p j}\right)-\left|p_{j}\right|\right)}{1-2\left|p_{j}\right| \cos \left(\Omega-\phi_{p j}\right)+\left|p_{j}\right|^{2}}- \\
& -\sum_{i=1}^{M} \frac{\left|c_{i}\right| \cdot\left(\cos \left(\Omega-\phi_{c i}\right)-\left|c_{i}\right|\right)}{1-2\left|c_{i}\right| \cos \left(\Omega-\phi_{c i}\right)+\left|c_{i}\right|^{2}}
\end{aligned}
$$

In the latter equation, $\left|c_{i}\right|,\left|p_{i}\right|$ are the modules of the zeros and the poles, and $\phi_{c i}, \phi_{p i}$ are their phases, respectively.

Finally, the absolute quadratic dispersion of the filter can be found using:

$$
D=-c \cdot\left(\frac{T}{\lambda}\right)^{2} \cdot 2 \pi \cdot \frac{d \tau_{n}}{d \Omega}
$$

where $c$ is the speed of light, $\lambda$ is the optical carrier's wavelength and:

$$
T=\frac{n_{e f} \cdot L_{T}}{c}
$$

The filter response is repeated with an spectral periodicity, or free spectral range (FSR), that equals $1 / T$. It would be desirable that the RR+SG filter fits the general framework of all-pass filters, in order to approximate an arbitrary phase response with unitary magnitude response, except for nonideal behaviors of photonic components. Examining equation (13) considering (14) and (15) we find that, unfortunately, the RR with a SG interferometer is not an all-pass filter. The consequence of this is a tradeoff between the quadratic dispersion reached and the magnitude distortion.

In Fig. 2 an example of design is considered for $K_{1}=0.3$ with a FSR equal to $25 \mathrm{GHz}$. The magnitude response in logarithmic scale $(\mathrm{dB})$ is shown in Fig. 2(a) versus optical frequency deviation $(\mathrm{GHz})$ with regards to the center frequency of the simulations, which equals $193.1 \mathrm{THz}$ (around $1553 \mathrm{~nm}$ ). In Fig. 2(b) the quadratic dispersion of the filter in $\mathrm{ps} / \mathrm{nm}$ is shown versus the same scale of optical frequency. By changing the coupling value $K_{2}$ the tuning of the filter is achieved, due to the fact that the phases of the conjugate pairs of zeros and poles increase while keeping their distance to the unit circle [14].
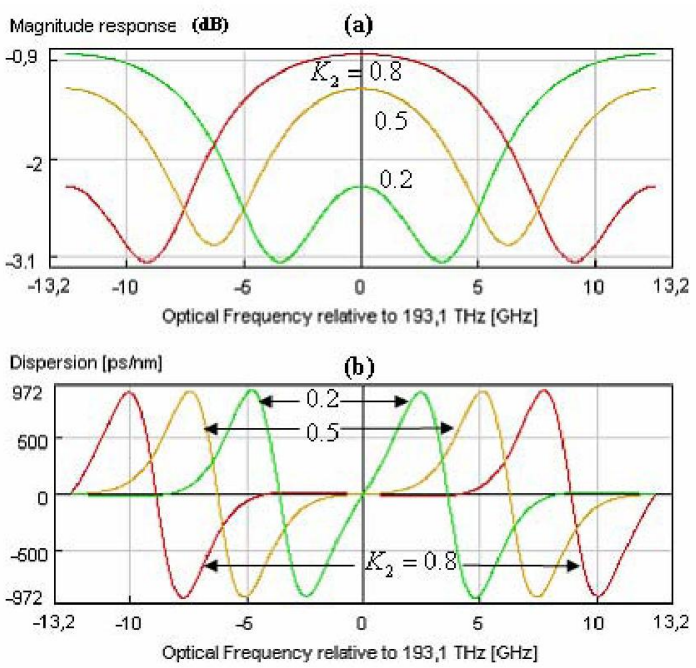

Fig. 2. Magnitude response versus frequency for $K_{I}=0.3, g=0.6$ (a) and quadratic dispersion (b) of a RR+SG filter with $F S R=25 \mathrm{GHz}$. $\gamma_{1}=\gamma_{2}=0.075, \mathrm{~g}=0.6(-4.4 \mathrm{~dB})$ 
As Fig. 2 shows, the $\mathrm{RR}+\mathrm{SG}$ filter has a symmetrical magnitude response with regards to the center frequency and anti-symmetrical dispersion shapes in each half of the FSR.

As $K_{2}$ increases, the dispersion lobes separate more from each other, leaving a zero dispersion range with a flat shape in the middle of the FSR, see Fig. 2(b). This quick return from the dispersion peaks to zero is a good property to take advantage from when combining the $\mathrm{RR}+\mathrm{SG}$ quadratic dispersion to other RR-based filters. Because the zeros and the poles have not inverse magnitudes, as it would happen for an all-pass filter, the design of Fig. 2 shows a magnitude distortion in frequency with maximum insertion loss of $3 \mathrm{~dB}$. For this $K_{1}$ value, in order to have a higher dispersion peak, see Fig. 2(b), it is needed to adjust the roundtrip losses closer to the resonance condition, which results in unwanted higher magnitude distortion.

In order to avoid the effect of the poles and to have a simpler design no optical amplification is considered. Additionally, the design point must be far away from resonance conditions, different for each zero when they are not complex conjugated.

\section{RR+SG BASED FILTERS AS DISPERSION COMPENSATION MODULES (DCM)}

In addition to the analysis of the quadratic dispersion of filters, there is a need for testing the behaviour of the configurations when noise and digital signals appear at the reception stages. Actually, for a fixed bit error rate in an optical transmission system, there is an inversely proportional relation between the bit rate and the transmission distance. In this section we investigate the effect of including a $R R+S G$ based filter (Fig. 3) at the reception stage of a simplified model of a backbone link.

Fig. 3 shows the group delay and quadratic dispersion of a compound design using a $2-\mathrm{RR}$ in series and a RR+SG filter connected to its output. Two lossless RR in series with coupling factors of 0.84 and 0.68 with relative phase shift of 0.7 rads were considered. The design parameters of the $\mathrm{RR}+\mathrm{SG}$ configuration are $K_{1}=0.3, K_{2}=0.8 \quad g=0.6$ and $\gamma_{1}=\gamma_{2}=0.075$, see Fig. 3 . The periodicity of all the stages is $25 \mathrm{GHz}$, and so is the FSR of the total configuration.

In order to test the performance of RR-based filters in an optical digital transmission system operating at high bit rates, a simplified model of the backbone link has been built using the VPI Photonics TM v. 7.01 software simulation engine. Results reported in [14] shows a power penalty enhancement between 3-4 $\mathrm{dB}$ when the filter is emplaced at the reception stage of the link. Comparisons with conventional dispersion compensating fiber DCF, modules have also been reported
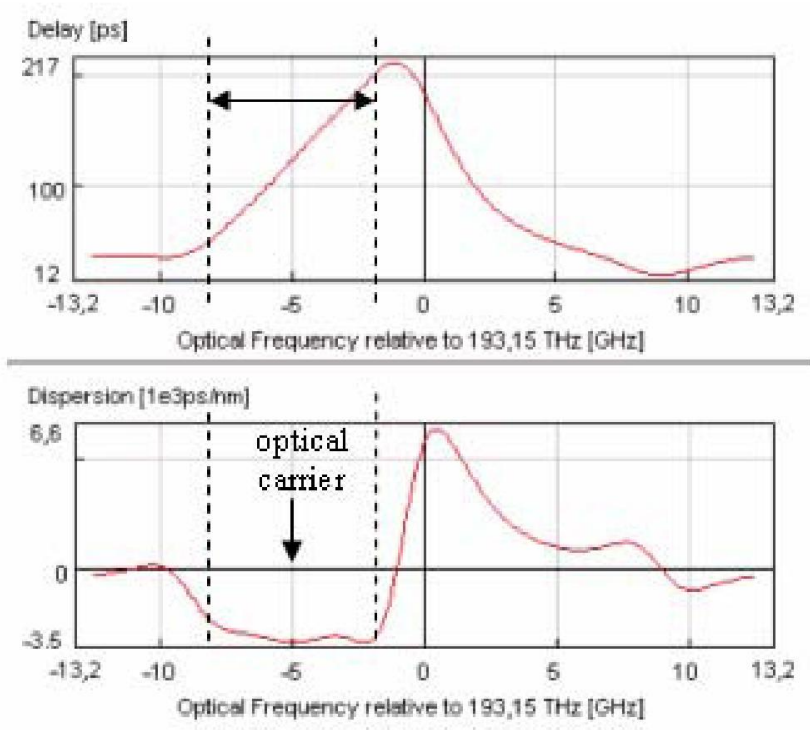

Fig. 3. Group delay (up) and quadratic dispersion (down) of a 2-RR with $\mathrm{RR}+\mathrm{SG}$ in series compound filter as a dispersion compensating module.

\section{RR TECHNOLOGY DESIGN}

The RR can be fabricated in integrated optics technology using different materials such as InGaAsP/InP [15], GaAs/AlGaAs, Silicon-On-Insulator (SOI) [16] and polymeric materials [17].

For these ultra-compact devices it is usually required an external trimming of the configuration to overcome the tolerance errors during the lithographic fabrication process. We have previously reported designs of switches made of RR in SOI technology and using liquid crystals as the control element [9], see Fig. 4.

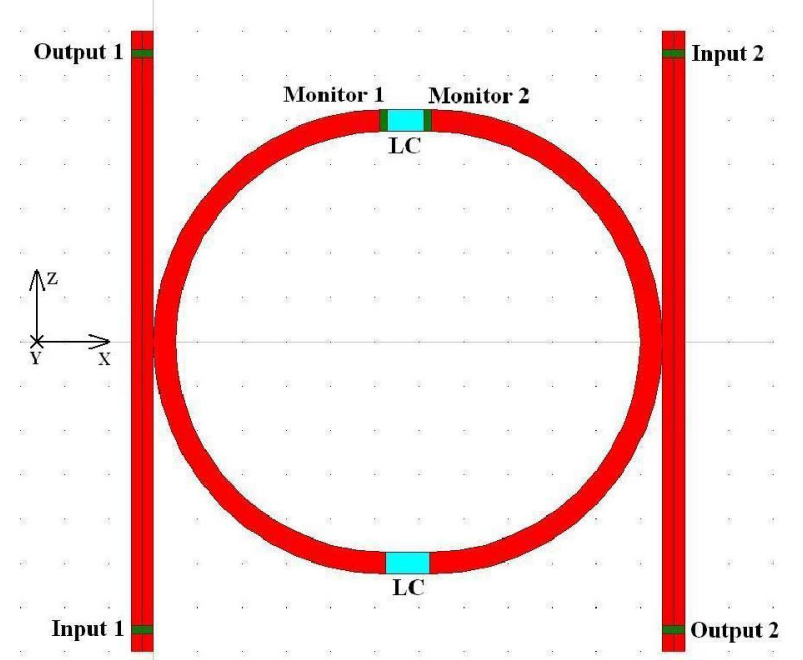

Fig. 4. Four-ports $\mu R R$ schematic using LC in the loop. 
The 2D prototypes consist of $\mu$ RR with 5 microns radius with slab $\mathrm{SiN}$ core waveguides of 0.5 microns width on $\mathrm{SiO}_{2}$ cladding. Using liquid crystals cells as adjusting elements, simulations with commercial software in the $1550 \mathrm{~nm}$ band show FSR around $4 \mathrm{THz}$ for LCs with $1 \mu \mathrm{m}$ length.

By increasing the RR radius to $300 \mu \mathrm{m}$ and using the effective refractive indexes of the waveguides corresponding to the polymeric technology [17], such as $n_{\text {core }}=1.582$ and $\mathbf{n}_{\text {cladding }}=1.409$, FSRs around $100 \mathrm{GHz}$ can be obtained, fitting the DWDM spectral grid thus allowing applications for the standard SONET/SDH communications networks.

\section{SUMMARY AND CONCLUSION}

The optical configuration consisting of a ring resonator with an internal Sagnac loop $(\mathrm{RR}+\mathrm{SG})$ has been studied for chromatic dispersion managing in backbone SMF links. A mirror model of the configuration has been considered to obtained closed-form formulae of its transfer function, group delay and quadratic dispersion. We have examined graphically the frequency tuning of the dispersion peaks by changing the coupling factor in the Sagnac loop, with no need for using phase-shifting techniques. The magnitude distortion restriction of the RR+SG configuration has also been analyzed.

Additionally, we have presented results of a RR+SG based filter as a dispersion compensating module (DCM). Simulations have shown a power penalty enhancement between 3-4 dB when the filter is emplaced at the reception stage of the link. Another advantage of the RR-based filters as DCM is the periodicity of the transfer function, thus fitting multi-channel DWDM systems with a large number of optical carriers with maximum change in chromatic dispersion around $300 \mathrm{ps} / \mathrm{nm}$ for a $200 \mathrm{~km}$ SMF link in the complete Cband $(1530-1560 \mathrm{~nm})$. Fabrication technology issues have also been considered.

\section{ACKNOWLEDGMENT}

This work was partially supported by Spanish CICYT (TEC2006-13273-C03-03-MIC), European project NoE EPhoton/One+, CAM (FACTOTEM-CM:S0505/ESP/000417), FENIS-CCG06-UC3M/TIC-0619.

\section{REFERENCES}

[1] Shyh-Lin Tsao, Hen-Wai Tsao, Yang-Han Lee, "Design of a SelfRouting Frequency Division Multiple Access (SR-FDMA) Network Using an Optical Ring Filter with or without Gain as a Router", JLT, Vol. 13, No.11, Nov. 1995.

[2] C. Vázquez, S. Vargas, J. M. S. Pena, "Design and Tolerance Analysis of a Router using an Amplified Ring Resonator and Bragg Gratings" Applied Optics, vol. 39, pp. 1934-1940, 2000.

[3] J.M. Senior, M.R. Handley, and M.S. Leeson. "Developments in Wavelength Division Multiple Access Networking". IEEE Communications Magazine, 28-36, December 1998.

[4] Otto Schwelb "Transmission, Group Delay, and Dispersion in SingleRing Optical Resonators and Add/Drop Filters-A Tutorial Overview" J. Lightwave Tech, vol. 22, 5, 2004, pp. 1380-1394.
[5] C. Vázquez, F. Hernández-Gil, M. López-Amo "Tunable Ring Resonator Filter for OFDM Transmission Systems", Microwave and Optical Technology Letters, Vol. 8, No. 6, Apr. 1995

[6] B. E. Little, et al. "Very High-Order microring resonator Filters for WDM Applications" IEEE Photonics Tech. Lett., vol16, no.10, pp. 2263-2265, 2004

[7] S. Dilwali and G.S. Pandian, "Pulse response of a fiber dispersion equalizing scheme based on an optical resonator", IEEE Photon. Techn. Lett. 4 (8), 942-944 (1992).

[8] J. E. Heebner and R. W. Boyd, "Enhanced all-optical switching by use of a nonlinear fiber ring resonator," Opt. Lett. 24, 847-849 (1999)

[9] C. Vázquez, P. C. Lallana, J. Montalvo, J.M. Sánchez Pena, A d'Alessandro, D. Donisi, "Switches and tunable filters based on ring resonators and liquid crystals" SPIE's International Symposium on Microtechnologies for the New Millennium 2007. Photonics and Optoelectronics, Maspalomas 2007

[10] C. K. Madsen, J. H. Zhao, Optical Filter Design and Analysis, A Signal Processing Approach, John Willey and Sons, New York, 1999.

[11] C.K. Madsen, J.A. Walker, J.E. Ford, K.W. Goossen, T.N. Nielsen and G. Lenz, "A tunable dispersion compensating MEMS all-pass filter", IEEE Photon. Techn. Lett. 12(6), 651-653 (2000).

[12] C. Vázquez, Salvador E. Vargas, J. M. Sánchez-Pena, "Sagnac loop in ring resonators for tunable optical filters", IEEE Journ. of Lightw. Technol. 23 (8), 2555-2567 (2005).

[13] C. Vázquez, S. Vargas, J.M.S. Pena and P. Corredera, "Tunable optical filters using compound ring resonators for DWDM", IEEE Photon. Techn. Lett. 15(8), 1085-1087 (2003).

[14] J. Montalvo, C. Vázquez "Ring resonator with an internal Sagnac loop for dispersion compensation in DWDM backbone networks" SPIE's International Symposium on Microtechnologies for the New Millennium 2007. Photonics and Optoelectronics, Maspalomas 2007.

[15] Uriel Levy Kyle Campbell, Alex Groisman, Shayan Mookherjea, Yeshaiahu Fainman, "On-chip microfluidic tuning of an optical microring resonator", Appl. Phys. Lett., 88, 111107 (2006).

[16] Prakash Koonath, Tejaswi Indukuri, and Bahram Jalali, "Monolithic 3D Silicon Photonics", J. Light. Tech. Lett., 24(4), 1796-1804 (2006).

[17] A. Maalouf, D. Bosc, F. Henrio, S. Haesaert, P. Grosso, I. Hardy, M Gadonna, "Polymer optical circuits technology for large-scale integration of passive functions", Integrated Optics, Silicon Photonics, and Photonic Integrated Circuits, edited by Giancarlo C. Righini, Proc. of SPIE Vol. 6183, 61831H (2006). 